Case Report

\title{
Postural Instability in a Young Dyslexic Adult Improved by Hebbian Pulse-width Modulated Lighting
}

\author{
Albert Le Floch ${ }^{1,2, \text { *, Samuel Henriat }}{ }^{3}$, Rosane Fourage ${ }^{4}$, Guy Ropars ${ }^{1, \text { * }}$ \\ ${ }^{1}$ Laser Physics Laboratory, University of Rennes 1, Rennes, France \\ ${ }^{2}$ Quantum Electronics and Chiralities Laboratory, Rennes, France \\ ${ }^{3}$ Clinic of Podiatry, Pontivy, France \\ ${ }^{4}$ Institute of Training in Podology, Ergotherapy and Kinesitherapy, Rennes, France
}

Email address:

albert.lefloch@laposte.net (A. Le Floch), guy.ropars@univ-rennes1.fr (G. Ropars)

${ }^{*}$ Corresponding author

\section{To cite this article:}

Albert Le Floch, Samuel Henriat, Rosane Fourage, Guy Ropars. Postural Instability in a Young Dyslexic Adult Improved by Hebbian Pulse-width Modulated Lighting. American Journal of Internal Medicine. Vol. 8, No. 6, 2020, pp. 267-273. doi: 10.11648/j.ajim.20200806.15

Received: October 14, 2020; Accepted: October 23, 2020; Published: November 4, 2020

\begin{abstract}
Background: Postural stability is linked to vision in everyone, since when the eyes are closed stability decreases by a factor of 2 or more. However, in persons with dyslexia postural stability is often deficient even when the eyes are open, since they show deficits in motor as well as specific cognitive functions. In dyslexics we have shown that abnormal symmetry between retinal Maxwell's centroid outlines occurs, perturbing the interhemispheric connections. We have also shown that pulse-width modulated lighting can compensate for this lack of asymmetry, improving the reading skills. Objective: As the postural stability and the vision are correlated, one may wonder if the excess of the postural instability recorded in a young adult with dyslexia can also be reduced by a pulse-width modulated light controlling the Hebbian synaptic plasticity. Method: Using a force platform we compared the postural responses of an observer without dyslexia with the responses of a subject with dyslexia, by measuring their respective standing postures with eyes open looking at a target in a room with either continuous or pulse lighting. Results: There was no effect of changing the lighting conditions on the postural control of the subject without dyslexia. However, we found that the postural stability of the subject with dyslexia which was actually impaired during continuous light, but was greatly improved when a $80 \mathrm{~Hz}$ pulsed light frequency was used. Importantly, the excursions of the surface area of the center of pressure on the force platform were reduced by a factor of 2.3. Conclusion: The postural instability in a dyslexic person can be improved by pulse-width modulated lighting.
\end{abstract}

Keywords: Neural Connectivity, Postural Control, Visual Interactions, Synaptic Plasticity, Dyslexia, Pulse Light Effect

\section{Introduction}

The role of vision in the maintenance of posture and the visual stabilization of posture has been observed for normal persons by many groups [1-4]. Postural sway measured using force platforms is commonly used to compare the human posture in quiet stance with eyes open and eyes closed for children and older people $[5,6]$. In these studies the postural fluctuations have been shown to increase when the eyes are closed, showing the important role of vision and neural connections in postural stability.

Developmental dyslexia is a complex specific learning difficulty that affects reading, writing, spelling and the development of literacy and language [7-9]. Moreover the problems faced by many dyslexic children are by no means confined to these difficulties [10]. The postural deficiency syndrome [11] and different impairments in motor skills [10, 12], balancing ability [13], and postural control [14-18] are often observed in dyslexic people and in children with developmental disorders $[19,20]$.

In dyslexic children and young adults, a whole-brain analysis of the connectivity using functional magnetic resonance has shown different disruptions of functional networks [21], with a lack of lateralization, namely in the left visual-word-form area [22] but also with highly significant differences in functional connectivity when compared to a control group. The use of the 
functional trans-cranial Doppler ultrasound method to directly access the cerebral asymmetry, has confirmed the reduction of the usual left-sided language lateralization in dyslexia [23]. The brain lateralization and the interhemispheric connectivity being closely linked to motor control and adaptation [24, 25], impairments in the connectivity in dyslexia result in different possible motor impairments including motor overflow [26-29], posture instability [14-18], balancing ability in children [13, 30] and in adults [31-33]. Indeed, brain activation during maintenance of standing postures in humans confirms that the visual cortex plays an important role [6,34]. The links between the primary visual and motor areas are complex and involve a series of cortico-cortical links. Moreover visual areas through the brain are connected to motor areas through the cerebellum which is strongly engaged in motor control including posture and balancing ability, while also playing a role in different cognition functions [35-38]. Unintentional motor impairments such as motor overflow with mirror movements of the hands and effects replicating primary reflex movements on specific reading difficulties in dyslexic children [39], also suggest weak cerebral asymmetry and weak brain lateralization in dyslexic people.

The lack of interhemispheric asymmetry linked to the Maxwell's centroid asymmetry in many dyslexic observers, has been shown to often include the simultaneous observation of veridical and mirror-images of letters like " $b$ " and "d" [40]. Since this study with students, we have observed similar results with many children and teenagers from 8 to 17 years old. Moreover, as a pulse-width modulation LED lamp, using Hebbian mechanisms, is able to erase the mirror-images, restoring the reading skills and providing the person with dyslexia with an assisted cerebral asymmetry [40], one may wonder if the muscular postural instabilities which often accompany the reading difficulties in these dyslexic observers, can also be reduced. It is the aim of this paper to investigate the effect of a pulse-width light modulation on the postural stabilization of a dyslexic observer. We compare the standing postural responses in binocular fixation tests of a dyslexic observer to those of a control observer without dyslexia, under first continuous light regime and then pulsed light regime using Hebbian mechanisms.

\section{Material and Methods}

\subsection{Participants}

The two volunteer male students G. L. and E. C. (22 years old) take the same scientific course in our University. They were aware of the purpose of the study. The informed consent was obtained from each participant after explaining the nature of the study. One of the students G. L., without dyslexia, has no specific reading problem, while the second student E. C. with dyslexia is assisted by the Health Center of the University which provides extra-time for the examinations. Both students have normal binocular vision. The entire investigation process was conducted according to the principles expressed in the Declaration of Helsinki.

\subsection{Optical Tests}

\subsubsection{The Asymmetry of Maxwell's Centroids}

Posture being linked to vision, we have first to investigate the presence or absence of imbalance in human vision, previously suspected by Hubel and Wiesel [41, 42]. An asymmetry has been detected at the center of the two foveas by comparing the two small blue cone-free areas which correspond to the so-called Maxwell centroids [40]. As the retinas are parts of the central nervous system, this asymmetry plays a crucial role in the connectivity and the lateralization of the brain. For each eye, the blue cone-free area is seen as a small dark zone on a blue background when fixating a bright white screen through a blue filter. Using a blue-green exchange filter in a so-called foveascope [40], the contrast can be optimized and the outlines of the Maxwell's centroid entoptic images recorded for each eye. To quantify the ellipticity of each blue cone-free area, we use an osculating ellipse and define the ellipticity $\varepsilon_{\mathrm{R}}$ and $\varepsilon_{\mathrm{L}}$ for the right and the left eye respectively. The ellipticity difference $\Delta \varepsilon=\varepsilon_{\mathrm{R}}-\varepsilon_{\mathrm{L}}$ defines the Maxwell's centroid asymmetry for each observer, and the sign of this difference determines the eye dominancy which is confirmed by the afterimage test (see below).

\subsubsection{Noise-activated Afterimages}

Eye dominancy being a hallmark of asymmetry in human vision, we had to determine the eye preference of the two observers. To avoid possible artefacts with the different sighting methods such as the distance of observations or the gaze angle, we use the noise-activated afterimage method [40], where the two eyes remain closed after a fixation by the two eyes on the same bright stimulus. The observer can look, for instance, at a black letter "c" on a bright screen. With his two eyes, the observer gazes at the "c" letter for about 10 seconds, then he closes his eyes and with his hands, blocks the 2 to $3 \%$ [40] of the diffused light passing through his closed eyelids which can act as noise. The two retinas are then equally imprinted. Now, by alternatively shifting the hands in front of each eye with a periodicity of about $2 \mathrm{~s}$, two noise-activated afterimages are seen successively, one for each eye. Hence, we have a differential method for comparing the activation in the brain of the same information encoded through each retina. The dominant eye corresponds directly to the brightest afterimage, i.e. to the eye with the strongest pathway to the brain.

The noise-activated afterimages have another interest. Indeed for many persons with dyslexia, the method allows us to directly see the mirror-image of letters which induces the crowding effect, detrimental to their reading skills. With the noise-activated afterimage test, the presence or the absence of extra mirror-images is easily detected. Moreover the technique allows us to directly observe the erasing of the mirror images using the Hebbian mechanisms in the case of a person with dyslexia, when they use a pulse-width modulation system during the fixation.

\subsection{Posture Measurements}

The performances of the two observers were recorded by a 
force platform (Médicapteurs) following the standard instructions [43-46]. To record the movement of the body's center of pressure with both eyes open, there is a circular target, i.e. a red laser spot of about $5 \mathrm{~mm}$ diameter on a wall that was situated $90 \mathrm{~cm}$ in front of the subject at eye level. The room had a normal level of illumination. However the lighting was provided by a LED panel which was electronically controlled. The system can work either in the usual continuous lighting regime (Figure 1a) or in a pulse-width modulation regime (Figure $1 \mathrm{~b}$ ), with a repetitive frequency which can be varied from 65 to $120 \mathrm{~Hz}$ [40].
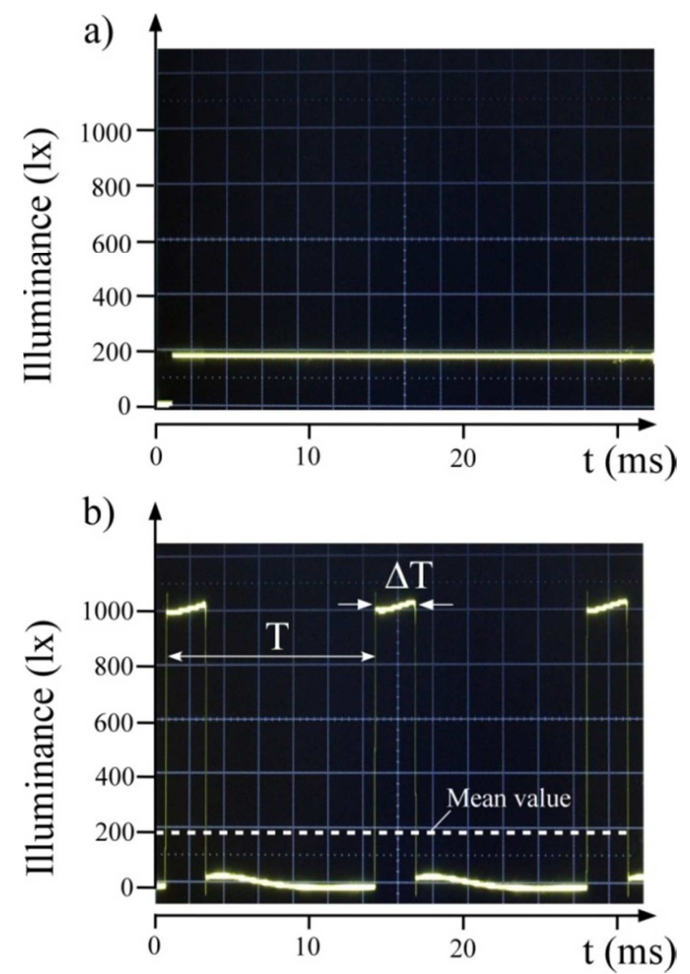

Figure 1. The two lighting regimes. a) Target illuminance in the continuous regime. b) Target illuminance in the $80 \mathrm{~Hz}$ pulsed regime, with a duty cycle $\Delta T / T=0.2$, to use the Hebbian mechanisms. The average illuminance is 200 lx in the two cases.

In the two regimes, the average illumination on the fixation target surface was $200 \mathrm{~lx}$. The observers stood barefoot on the force platform in a position with feet abducted at $30^{\circ}$, heels separated by $5 \mathrm{~cm}$, and their arms hanging loosely by their sides [17, 43-47]. The center of pressure sway signals and the latero-lateral and anterior-posterior signals were recorded with a sampling rate of $100 \mathrm{~Hz}$ for 30 s trial recordings. Three trials are repeated for each situation. For each participant the binocular fixation was performed first under the continuous lighting regime, then under the pulsed regime.

\section{Results}

\subsection{Optical Tests}

\subsubsection{Case of a Young Adult Without Dyslexia}

Figure 2 shows the standard vision recordings of the young adult without dyslexia. a) Maxwell's centroid outlines
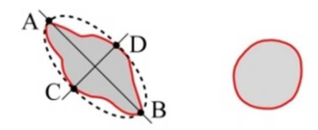

$$
\varepsilon_{\mathrm{L}} \simeq 0.5 \quad \varepsilon_{\mathrm{R}} \simeq 1
$$

b) Noise-activated afterimages

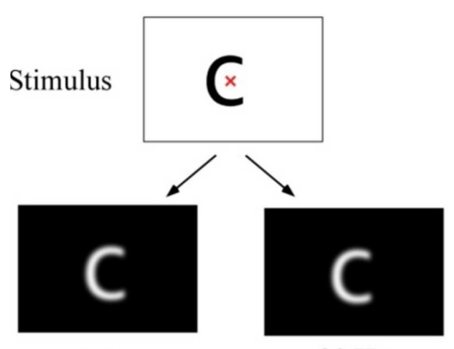

CW

$80 \mathrm{~Hz}$

Figure 2. Maxwell's centroid outlines and afterimages for a young adult without dyslexia. a) The asymmetry of the Maxwell's centroid outlines: here $\Delta \varepsilon=\varepsilon_{R}-\varepsilon_{L} \approx+0.5$. The quasi-circular right-eye outline for this observer corresponds to the dominant eye which is confirmed by the brighter noise-activated afterimage of his right eye. b) The observed afterimages of a "c" stimulus. The afterimages are unchanged with a continuous laboratory lighting or with an $80 \mathrm{~Hz}$ pulse-width modulated laboratory lighting.

First, the outlines of the Maxwell's centroids he observed (Figure 2a) show an asymmetry $\Delta \varepsilon=\varepsilon_{\mathrm{R}}-\varepsilon_{\mathrm{L}} \approx+0.5$. The eye preference of this observer is fixed by the quasi-circular outline in his right eye. This eye preference is in agreement with the brighter noise-activated afterimage he sees with his right eye. Second, either in the continuous lighting regime or in the $80 \mathrm{~Hz}$ pulse lighting regime, the binocular afterimage of the "c" stimulus remains unchanged (Figure 2b).

\subsubsection{Case of a Young Adult with Dyslexia}

The corresponding results for the young adult with dyslexia are reported in Figure 3.

a) Maxwell's centroid outlines

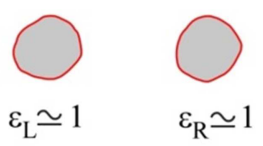

b) Noise-activated afterimages

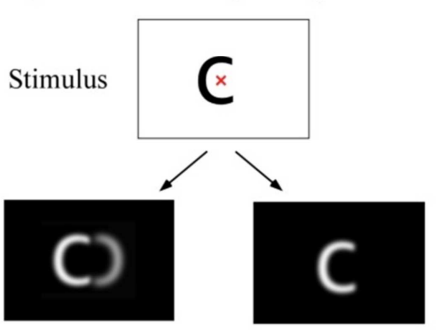

CW

$80 \mathrm{~Hz}$

Figure 3. Maxwell's centroid outlines and afterimages for a young adult with dyslexia. a) The seeming symmetrical outlines. The two quasi-circular outlines correspond for this observer to their two similar noise-activated afterimages and induce a no-dominant eye status. b) The observed afterimages of a " $c$ " stimulus. The afterimage in the continuous regime shows the coexistence of the primary image "c " and of the weaker mirror image " $\supset$ ". In the $80 \mathrm{~Hz}$ pulsed regime, with a duty cycle of 0.2 , the mirror-image is cancelled using the Hebbian mechanisms. 
Here, the asymmetry $\Delta \varepsilon=\varepsilon_{\mathrm{R}}-\varepsilon_{\mathrm{L}}$ between the two Maxwell's centroids is $\Delta \varepsilon \approx 0$ (Figure 3a). The two quasi-circular outlines correspond to an undetermined dominancy which is confirmed by the equal brightness of the afterimages seen by the two eyes of the observer. In contrast of the case of the non-dyslexic adult, the afterimage often seen by observers with dyslexia in the usual continuous lighting regime is the signature of a too high interhemispheric symmetry, i.e. a too weak lateralization in the brain [40]. Indeed here, the symmetric mirror-image of the stimulus "c" projected through the callosum between the two hemispheres is not weakened enough, and coexists with the veridical afterimage, perturbing the observer's reading skills (left part of Figure $3 \mathrm{~b}$ ). However, as described in ref. 40, when the afterimages are observed using a $80 \mathrm{~Hz}$ pulse light during the fixation, the extra mirror-image is erased (right part of Figure $3 b$ ), and the observed afterimage is identical to that seen by the observer without dyslexia. The Hebbian mechanisms governing the synapse plasticity contribute to the weakening of the mirror-image, inducing an assisted asymmetry and strengthening lateralization in the brain. The cognitive functions such as reading are improved and we can also now investigate the respective postural effects for the two observers in the continuous or pulsed light regimes.

\subsection{Postural Measurements}

\subsubsection{Case of a Young Adult Without Dyslexia}

On Figure $4 \mathrm{a}$ the latero-lateral and the anterior-posterior performances of the young adult without dyslexia were recorded for 30 s trials.

\section{a) $\mathrm{CW}$ regime}
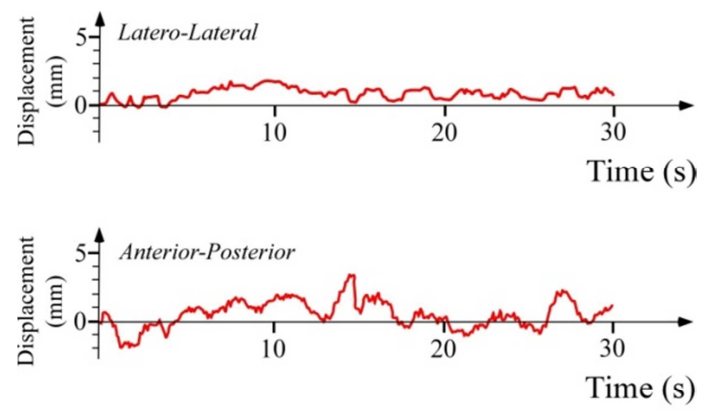

b) $80 \mathrm{~Hz}$ regime

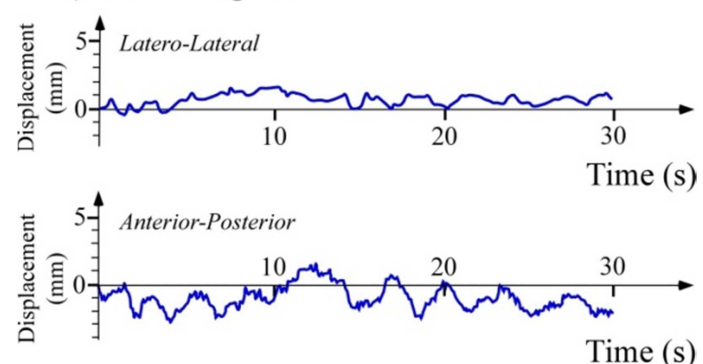

Time (s)

Figure 4. Force platform results for a young adult without dyslexia. a) Latero-lateral and anterior-posterior variations under $\mathrm{CW}$ laboratory lighting. The surface of the stabilogram is $10 \mathrm{~mm}^{2}$. b) Same results under 80 $\mathrm{Hz}$ pulse laboratory lighting. The results are unchanged (here the surface of the stabilogram is $8 \mathrm{~mm}^{2}$ ).
Following the standard instructions three trials are repeated for each situation. The respective variations were rather weak. No appreciable changes were observed in the $80 \mathrm{~Hz}$ pulsed light regime (Figure 4b). The surface of the center of pressure was $10 \pm 2 \mathrm{~mm}^{2}$ continuous regime, and $8 \pm 2 \mathrm{~mm}^{2}$ in the pulsed regime.

\subsubsection{Case of a Young Adult with Dyslexia}

The corresponding measurements for the young adult with dyslexia are reported in Figure 5.

\section{a) $\mathrm{CW}$ regime}

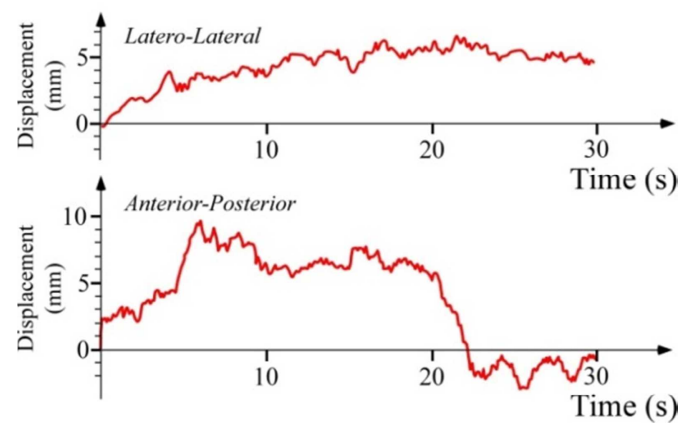

b) $80 \mathrm{~Hz}$ regime

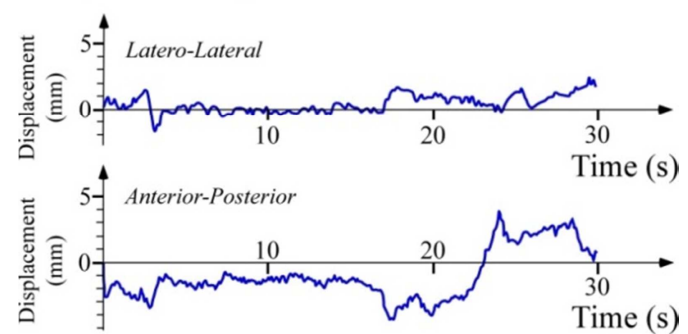

Figure 5. Force platform results for a young adult with dyslexia. a) Latero-lateral and anterior-posterior variations under continuous lighting. The results show a postural deficiency, which are in agreement with the 66 $\mathrm{mm}^{2}$ surface for the centre of pressure. b) Same results under $80 \mathrm{~Hz}$ pulsed lighting. Both the latero-lateral and the anterior-posterior variations are reduced by a factor 1.5 and the surface $\left(28 \mathrm{~mm}^{2}\right)$ is reduced by a factor 2.3 thanks to the Hebbian mechanisms, showing the neural plasticity.

\section{$120 \mathrm{~Hz}$ regime}
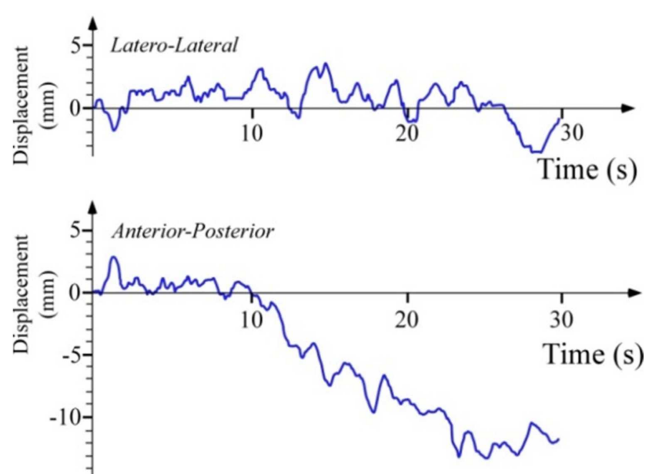

Figure 6. Force platform results for the young adult with dyslexia under 120 Hz pulse lighting. Both the latero-lateral and the anterior-posterior variations are increased by a factor 1.3 and 1.2 respectively and the surface $\left(100 \mathrm{~mm}^{2}\right)$ is increased by a factor 1.5, when compared to the continuous lighting corresponding here to the strengthening of the Hebbian synaptic plasticity. Note that the surface for the $120 \mathrm{~Hz}$ pulsed lighting is 3.5 times larger than the surface for the $80 \mathrm{~Hz}$ pulsed lighting. 
In the continuous lighting regime (Figure 5a) the latero-lateral and anterior-posterior oscillations were significantly larger compared to those of the first observer, by approximately a factor 3 . The surface area of the center of pressure reached $66 \pm 7 \mathrm{~mm}^{2}$. However, interestingly, in the presence of the $80 \mathrm{~Hz}$ pulsed lighting (Figure 5b), the latero-lateral and anterior-posterior oscillations were reduced by a factor 1.5 and the surface area of the center of pressure $\left(28 \pm 4 \mathrm{~mm}^{2}\right)$ was reduced by a factor 2.3 . The same tests repeated six months later confirm the results. Moreover, according to the Hebbian mechanisms governing the synaptic plasticity [48], for a non-matched modulation frequency of the lighting like $120 \mathrm{~Hz}$, the stability was not improved as at 80 $\mathrm{Hz}$, but was worsened.

The displacement variations were increased by a mean value of 1.25 , and the surface was increased by a factor of 1.5 (Figure 6). The stability measurements for the control observer remained unchanged irrespective of the lighting frequency.

\section{Discussion}

The postural performances for a person with a normal condition, although strongly linked to vision, remained unchanged in binocular vision fixation of a target whatever the continuous or pulsed regime of the lighting. By contrast the postural instabilities which persist for the young adult with dyslexia, even with the eyes open, were shown to be controlled by the lighting regime which also controls specific cognitive functions.

The strengthening of the asymmetry and of the lateralization via the Hebbian mechanisms [40, 48] exploiting the neural plasticity which permits the interhemispheric mirror-images in the reading tasks for a person with dyslexia to be erased, could also reduce the postural instabilities. This suggests that the visual information reaches the cerebellum which seems to be the most important link between visual and motor areas [49]. The brain cortex is known to be bidirectionally connected to the cerebellum through multiple circuits. Standing being a process of feedback control, involves continuous compensatory adjustments of the musculature. Indeed, neurons of the visual cortex communicate through extensive axonal projections targeting highly diverse areas [50], and posture cells have reciprocal connections to various cortical areas including the visual cortex $[51,52]$. These neuronal bonds are likely to be involved in our postural observations.

\section{Conclusion}

To conclude, vision and posture deficits appear intrinsically linked in the brain of the observer with dyslexia. The two retinas, as parts of the central nervous system, send a large mass of information to the cortex which processes the information to be transferred to other brain areas and the rest of the body. The stabilometric experiments with the young adult with dyslexia, suggest that the vision controls the posture. The lack of asymmetry in the young dyslexic adult can then be optically assisted via the Hebbian mechanisms governing the synaptic plasticity, improving both the specific cognitive functions like reading, and by cascading effects improving the motor functions like posture for a $80 \mathrm{~Hz}$ pulse modulated lighting. We hope that these postural results will be observed by other groups. As among the different sensory inputs to the brain vision seem to play a crucial role, the pulse-width modulated light effect could perhaps also be observed in the developmental coordination disorders such as dyspraxia and attention deficit hyperactivity disorder. Further studies will be needed for an extension to the different cases and perhaps will lead to useful applications.

\section{Acknowledgements}

The authors wish to thank the two students G. L. and E. C. for their kind participation, and B. Mitchell and K. Dunseath for the proofreading of the manuscript.

\section{References}

[1] W. M. Paulus, A. Straube, T. Brandt, Visual stabilization of posture, Physiological stimulus characteristics and clinical aspects, Brain 107 (1984) 1143-1163.

[2] T. T. Lê, Z. Kapoula, Role of ocular convergence in the Romberg quotient, Gait Posture 27 (2008) 493-500.

[3] D. N. Lee, The optic flow field: the foundation of vision, Philos Trans R Soc Lond B Biol Sci. 290 (1980) 169-179.

[4] E. E. Hansson, A. Beckman, A. Håkansson, Effect of vision, proprioception, and the position of the vestibular organ on postural sway, Acta Otolaryngol. 130 (2010) 1358-1363. DOI: 10.3109/00016489.2010.498024.

[5] M. Piirtola, P. Era, Force platform measurements as predictors of falls among older people - a review, Gerontology 52 (2006) $1-16$.

[6] M. Wade, G. Jones, The Role of Vision and Spatial Orientation in the Maintenance of Posture, Physical therapy 77 (1997) 619-628.

[7] T. Vidyasagar, Neural underpinnings of dyslexia as a disorder of visuo-spatial attention, Clinical \& experimental optometry 87 (2004) 4-10. https://doi.org/10.1111/j.14440938.2004.tb03138.x.

[8] J. Stein, The magnocellular theory of developmental dyslexia, Dyslexia 7 (2001) 12-36.

[9] M. Habib, The neurological basis of developmental dyslexia: An overview and working hypothesis, Brain 123 (2001) 2373-2399. DOI: 10.1093/brain/123.12.2373.

[10] R. I. Nicolson, A. Fawcett, P. Dean, Developmental dyslexia: The cerebellar deficit hypothesis, Trends in Neurosciences 24 (2001) 508-511. DOI: 10.1016/S0166-2236(00)01896-8.

[11] H. Martins Da Cunha, Syndrome de déficience posturale, Actualité en rééducation fonctionnelle et en réadaptation. $4 \mathrm{e}$ série. Paris: Masson (1979) 27-31. 
[12] J. Barela, P. de Freitas, A. Viana, M. Razuk, Dyslexia and the Integration of Sensory Cues into Motor Action, Psychology 5 (2014) 51107. DOI: 10.4236/psych.2014.516192.

[13] C. Stoodley, A. Fawcett, R. Nicolson, J. Stein, Impaired balancing ability in dyslexic children, Experimental brain $\begin{array}{lllll}\text { research } & 167 & (2005) & 370-380 . & \text { DOI: }\end{array}$ 10.1007/s00221-005-0042-x.

[14] T. Pozzo, P. Vernet, C. Creuzot-Garcher, F. Robichon, A. Bron, P. Quercia, Static postural control in children with developmental dyslexia, Neuroscience letters 403 (2006) 211-215. DOI: 10.1016/j.neulet.2006.03.049.

[15] Z. Kapoula, M. Pia Bucci, Postural control in dyslexic and non-dyslexic children, Journal of neurology 254 (2007) 1174-1183. DOI: 10.1007/s00415-006-0460-0.

[16] S. Vieira, P. Quercia, C. Michel, T. Pozzo, F. Bonnetblanc, Cognitive demands impair postural control in developmental dyslexia: A negative effect that can be compensated, Neuroscience letters 462 (2009) 125-129. DOI: 10.1016/j.neulet.2009.06.093.

[17] M. Pia Bucci, E. Bui, C.-L. Gerard, The Effect of a Stroop-like Task on Postural Control in Dyslexic Children, PloS one. 8. (2013) e77920. DOI: 10.1371/journal.pone.0077920.

[18] F. Ramus, E. Pidgeon, U. Frith, The Relationship Between Motor Control and Phonology in Dyslexic Children, Journal of child psychology and psychiatry, and allied disciplines 44 (2003) 712-722. DOI: 10.1111/1469-7610.00157.

[19] A. Kirby, D. A. Sugden, Children with developmental coordination disorders, J R Soc Med. 100 (2007) 182-186. DOI: 10.1258/jrsm.100.4.182.

[20] A. Gaddis, K. S. Rosch, B. Dirlikov, D. Crocetti, L. MacNeil, A. D. Barber et al., Motor overflow in children with attention-deficit/hyperactivity disorder is associated with decreased extent of neural activation in the motor cortex, Psychiatry Res. 30 (2015) 488-495. DOI: 10.1016/j.pscychresns.2015.08.001.

[21] E. Finn, X. Shen, J. Holahan, D. Scheinost, C. Lacadie, X. Papademetris, et al., Disruption of Functional Networks in Dyslexia: A Whole-Brain, Data-Driven Analysis of Connectivity, Biological psychiatry 76 (2013) 397-404. DOI: 10.1016/j.biopsych.2013.08.031.

[22] S. Dehaene, L. Cohen, The unique role of the visual word form area in reading, Trends in cognitive sciences 15 (2011) 254-262. DOI: 10.1016/j.tics.2011.04.003.

[23] D. Bishop, Cerebral Asymmetry and Language Development: Cause, Correlate, or Consequence?, Science 340 (2013) 1230531. DOI: $10.1126 /$ science. 1230531.

[24] N. Tzourio-Mazoyer, Intra- and Inter-hemispheric Connectivity Supporting Hemispheric Specialization, In: H. Kennedy, D. C. Van Essen, Y. Christen, editors. Micro-, Mesoand Macro-Connectomics of the Brain [Internet]. Cham $(\mathrm{CH})$ : Springer; 2016. Available from: https://www.ncbi.nlm.nih.gov/books/NBK435764/doi: 10.1007/978-3-319-27777-6_9.

[25] P. K. Mutha, K. Y. Haaland, R. L. Sainburg, The effects of brain lateralization on motor control and adaptation, J Mot Behav. $44 \quad$ (2012) 455-469. DOI: 10.1080/00222895.2012.747482.
[26] S. E. Parlow A Closer Look at Motor Overflow in Dyslexic Children. [Washington, D. C.], (1991). Distributed by ERIC Clearinghouse, https://eric.ed.gov/?id=ED333673.

[27] K. Hoy, P. Fitzgerald, J. L. Bradshaw, C. Armatas, N. Georgiou-Karistianis, Investigating the cortical origins of motor overflow, Brain Res. Brain Res. Rev. 46 (2004) 315-327. DOI: 10.1016/j.brainresrev.2004.07.013.

[28] E. D'Agati, L. Casarelli, M. Bernarda, A. Pasini, Overflow movements and white matter abnormalities in ADHD, ProgNeuropsychopharmacol Biol Psychiatry 34 (2010) 441-445. DOI: 10.1016/j.pnpbp.2010.01.013.

[29] P. Addamo, M. Farrow, K. Hoy, J. L Bradshaw, N. Georgiou-Karistianis, The effects of age and attention on motor overflow production-A review, Brain Res Rev 54 (2007) 189-204. DOI: 10.1016/j.brainresrev.2007.01.004.

[30] A. Fawcett, R. I. Nicolson, Dyslexia: The Role of the Cerebellum, in book: Dyslexia in Context: Research, Policy and Practice, (2008). 13-22. DOI: 10.1002/9780470777916.ch2.

[31] J. L. Needle, A. J. Fawcett, R. I. Nicholson, Balance and dyslexia: An investigation of adults' abilities, European Journal of Cognitive Psychology 18 (2006) 909-936. https://doi.org/10.1080/09541440500412304.

[32] R. L. Brookes, S. Tinkler, R. I. Nicholson, A. J. Fawcett, Striking the right balance: Motor difficulties in children and adults with dyslexia, Dyslexia 16 (2010) 358-373. http://dx.doi.org/10.1002/dys.420.

[33] M. Patel, M. Magnusson, D. Lush, S. Gomez, P. A. Fransson, Effects of dyslexia on postural control in adults, Dyslexia 16 (2010) 162-174.

[34] Y. Ouchi, H. Okada, E. Yoshikawa, S. Nobezawa, M. Futatsubashi, Brain activation during maintenance of standing postures in humans, Brain 122 (1999) 329-338.

[35] F. Horak, Postural orientation and equilibrium: What do we need to know about neural control of balance to prevent falls? Age and ageing 35 (2006) ii7-ii11. DOI: 10.1093/ageing/afl077.

[36] R. L. Buckner, The cerebellum and cognitive function: 25 years of insight from anatomy and neuroimaging, Neuron. 80 (2013) 807-815. DOI: 10.1016/j.neuron.2013.10.044.

[37] R. Chiba, K. Takakusaki, J. Ota, A. Yozu, N. Haga, Human upright posture control models based on multisensory inputs; in fast and slow dynamics, Neuroscience research. 104 (2016) 96-104. DOI: 10.1016/j.neures.2015.12.002.

[38] J. D. Schmahmann. The cerebellum and cognition, $\begin{array}{llll}\text { Neuroscience } & \text { Letters } & 688 & \text { (2019) }\end{array}$ https://doi.org/10.1016/j.neulet.2018.07.005.

[39] M. McPhillips, P. G. Hepper, G. Mulhern, Effects of replicating primary-reflex movements on specific reading difficulties in children: A randomised, double-blind, controlled trial, Lancet. $355 \quad(2000) \quad 537-41 . \quad$ DOI: 10.1016/S0140-6736(99)02179-0.

[40] A. Le Floch, G. Ropars, Left - Right asymmetry of the Maxwell spot centroids in adults without and with dyslexia, Proceedings of the Royal Society B: Biological Sciences 284 (2017) 20171380. DOI: 10.1098/rspb.2017.1380. 
[41] D. H. Hubel, Eye, brain, and vision. New York, NY: Scientific American Library (1988).

[42] D. H. Hubel, T. N. Wiesel, Brain and visual perception. New York, Oxford University Press, Inc (2005)

[43] T. S. Kapteyn, W. Bles, C. Njiokiktjien, L. Kodde, C. Massen, \& J. M. F. Mol, Standardisation in platform stabilometry being part of posturography, Agressologie 24 (1983) 321-326

[44] F. Scoppa, R. Capra, M. Gallamini, R. Shiffer, Clinical stabilometry standardization Basic definitions - Acquisition interval - Sampling frequency, Gait \& posture 37 (2012) 290-292. DOI: 10.1016/j.gaitpost.2012.07.009.

[45] M. Yamamoto, K. Ishikawa, M. Aoki, K. Mizuta, Y. Ito, M. Asai et al., Japanese standard for clinical stabilometry assessment: Current status and future directions, Auris Nasus Larynx 45 (2018) 201-206. DOI: 10.1016/j.anl.2017.06.006.

[46] N. Pinsault, N. Vuillerme, Test-retest reliability of centre of foot pressure measures to assess postural control during unperturbed stance, Medical engineering \& physics 31 (2008) 276-286. DOI: 10.1016/j.medengphy.2008.08.003.
[47] P. M. Gaget, G. Biggo, La mesure en Posturologie, Institut de Posturologie (http://ada-posturologie.fr/MesureEnPosturologie.htm), Paris.

[48] D. O. Hebb, The organization of behavior: neuropsychological theory. New York: Wiley (1949).

[49] M. Glickstein, How are visual areas of the brain connected to motor areas for the sensory guidance of movement? Trends Neurosci. 23 (2000) 613-617.

[50] Y. Han, J. M. Kebschull, R. Campbell, D. Cowan, F. Imhof, A. M. Zador, et al. The logic of single-cell projections from visual cortex, Nature 556 (2018) 51-56. DOI: 10.1038/nature26159.

[51] P. Brotchie, R. A. Andersen, L. Snyder, S. J. Goodman, Head position signals used by parietal neurons to encode locations of visual stimuli, Nature 375 (1995) 232-235. DOI: $10.1038 / 375232 \mathrm{a} 0$.

[52] B. Mimica et al., Efficient cortical coding of 3D posture in freely behaving rats, Science 362 (2018) 584-598. DOI: https://doi.org/10.1101/307785. 